\title{
Research on the Application of Intelligent Calibration Device for Nuclear Power Plants Based on Wireless Sensor Technology
}

\author{
http://dx.doi.org/10.3991/ijoe.v12i05.5731 \\ Zhe Yang and Cheng Yang \\ Suzhou Nuclear Power Research Institute, Shenzhen, Guangdong, China
}

\begin{abstract}
Wireless technology possesses distinct technical and economic advantages in the design, construction, and operations management of nuclear power plants. To improve the level of autonomy of nuclear power plants, we discuss the research on the international system of developing wireless technology applications for use in nuclear power plants. Further studies have enabled the extensive use of wireless technology in China's nuclear power industry. The application of the proposed wireless sensor technology in nuclear power plants is based on reliability, environmental adaptability, electromagnetic compatibility, scalability, and maintainability requirements. Accordingly, the application will be a gradual process but wireless sensor technology needs to evolve continuously to meet these requirements. This study is designed to implement a thermal instrumentation calibration device using a Windows $C E$ embedded operating system as the core of the test procedures in accordance with the relevant requirements. We also maximize the considerable reliability of an embedded operating system in the traditional areas of metering industry. Results show that in using wireless technology in nuclear power plant, the general thermal instrumentation calibration application of an intelligent device system is low, data accuracy is high, and the process is stable.
\end{abstract}

Index Terms-Intelligent calibration, Nuclear power, Thermal instrumentation, Wireless sensor networks

\section{INTRODUCTION}

The rapid development of nuclear power plants has resulted in the increasing seriousness of addressing the problems related to these power plants. Thus, formulating an improved method to solve such problems is necessary. In particular, the use of wireless sensors possesses immense advantage compared with conventional methods. For example, the use of these sensors in nuclear power plants can reduce costs immensely. In recent years, using wireless sensors has achieved good results in several nuclear power plants. The thermal instrumentation industry market demand is increasing rapidly, thereby continuously boosting demand for thermal instrumentation calibration devices. Thermal instrumentation calibration devices with purely manual operation are computer controlled. The objective of this study is to design a new thermal instrumentation calibration device that can resolve the maximum limit of the problems of the current version of these devices. This device uses a professional embedded system to replace the traditional, general purpose computer, as well as maximizes the embedded and highly specialized computer technology. A systematic verification device hardware is designed to meet the requirements of reducing production costs. Thus, the development of a new generation of thermal verification device can humanize and professionalize the process of controlling nuclear power plants. Accordingly, we present a suite of algorithms for the self-organization of wireless sensor networks, in which several static nodes with highly constrained energy resources exist [1]. A wireless sensor network (WSN) has important applications, such as remote environmental monitoring and target tracking. Such applications have been enabled by the recent availability of sensors that are considerably small, inexpensive, and intelligent [2]. One of the fundamental issues in networks is coverage, thereby reflecting that a network is monitored or tracked by sensors. [3]. Wireless networks, in which numerous resourcelimited nodes are linked via low bandwidth wireless radios, have been the focus of exhaustive studies during the past few years [4]. Structural monitoring, which is the collection and analysis of structural response to ambient or forced excitation, is an important application of network-embedded sensing with significant commercial potential [5].

\section{STATE OF THE ART}

In industrial production, a thermal instrumentation calibration device with purely manual operation becomes the present computer-controlled operation. In the conventional verification process, the electricity is switched manually to standard thermocouple at different test points. It is the subject of the thermocouple thermoelectric power value and then records the results sheet. The verification steps entirely relying on manual operation are comprehensive but labor-intensive, involve extensive testing to calculate the amount of red tape, and inevitably result in human error. This scenario requires the operator to have considerable experience and efficiency in utilizing this method. After the 1990s, the popularity and gradual emergence of the general purpose computer as the host computer has resulted in the development of an intelligent thermal instrumentation calibration system. The original system requires manual operation on the domestic market that is immensely streamlined by a dedicated software program. This system has an extensive communication among its various components. The recording process can be sent to the host computer for integrated treatment. Moreover, the testing and calibrating efficiency has been considerably improved because accuracy and repeatability are guaranteed. However, many problems still exist in the thermal 
upgrading process verification equipment. These problems are significantly prominent in two aspects. First, the computer has become a decisive factor in the entire device and involves many unpredictable problems, such as operating system instability, conflict between software, and computer viruses. If the factory is not used for short-term repair, then it is bound to cause considerable economic losses to the producer in terms of the entire system maintenance. Repair and post-services have also caused enormous difficulties. Therefore, the introduction of computer technology provides convenience to users but causes an increase in equipment cost and maintenance workload to the manufacturers as well. Second, the modular thermal verification equipment provides a systematic, professional, and qualitative leap from that used in the past. However, within the limits of the R\&D capabilities of domestic manufacturers, this device still lacks a completely systematic and professional design. Consequently, several key instruments still need outsourcing. The application of the test device merely includes a limited component of this type of instrument functions. Therefore, this immature design causes tremendous waste. The significance of the current research lies in the design of a new thermal instrumentation calibration device. This device can address the current maximum limit of thermal instrumentation calibration device problems using professional embedded systems to replace the traditional general purpose computer. The proposed device uses embedded computer technology that is highly specialized, reliable, powerful, flexible, and convenient in the traditional areas of the metering industry (See figure 1, 2 ). Furthermore, the systematic verification device hardware is designed to meet the requirements of reducing production costs.

\section{METHODOLOGY}

\section{A. Wireless Sensor Applications in Nuclear Power Plants}

Wireless sensor is already used in the application of wireless technology in nuclear power plants, such as in the areas of voice, video, remote environmental monitoring, storage management, and identification, among others. However, the control, protection, and monitoring by the sensors are rarely applied mainly because of electromagnetic compatibility and data security. Regarding electromagnetic compatibility, the wireless transmission system may affect the normal operation of various equipment, including other strong electromagnetic interference transmission equipment, in the nuclear power plant. In terms of data security, wireless transmission systems are more susceptible to inadvertent or deliberate intrusion of air than wired systems, thereby affecting normal data transmission [6] (See figure 3).

\section{B. Nuclear Power Plants Using Wireless Sensor Requirements and Response Measures}

The application of a wireless transmission network in nuclear power plants must be considerably reliable, thereby requiring various appropriate measures (See figure 4), such as anti-interference ability of the coding, modulation technology, efficient network topology control, and secure network protocols [7]. The current mainstream wireless sensor transmission uses BSPK, QSPK, and other coding methods to enhance immunity; uses spread spectrum communications and data encryption to improve data security; and introduces network collision avoidance and retransmission mechanisms to ensure normal network transmission.

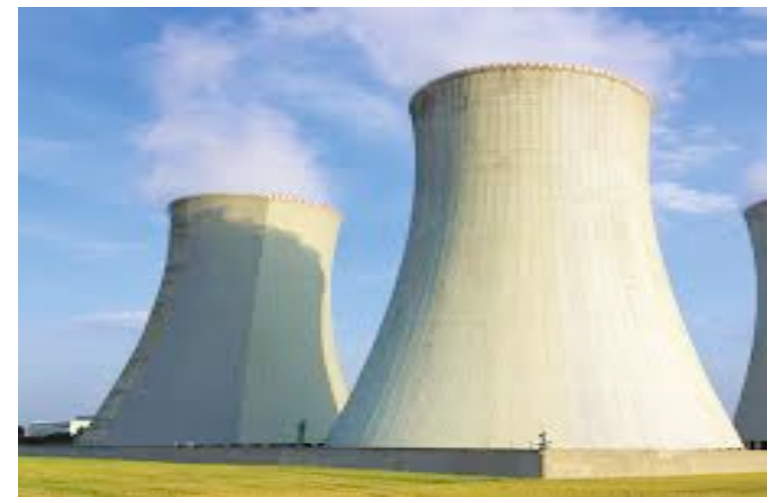

Figure 1. nuclear power plants

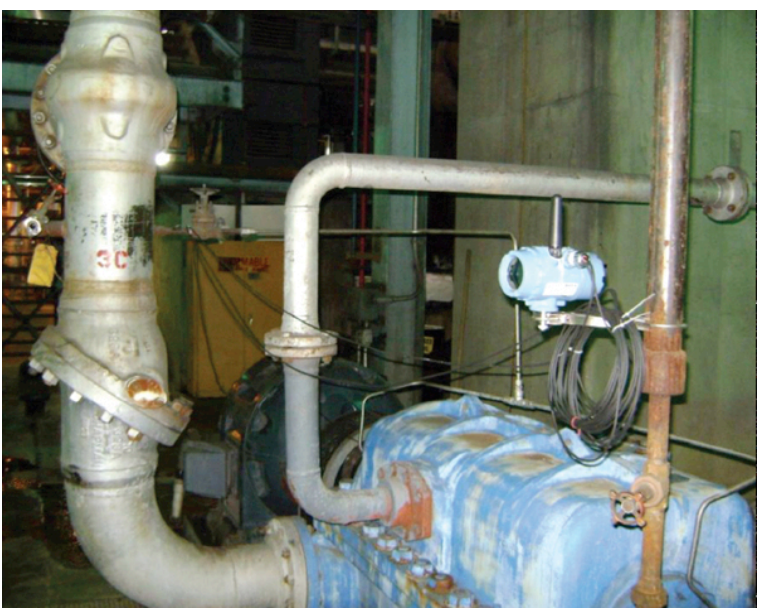

Figure 2. nuclear power plants

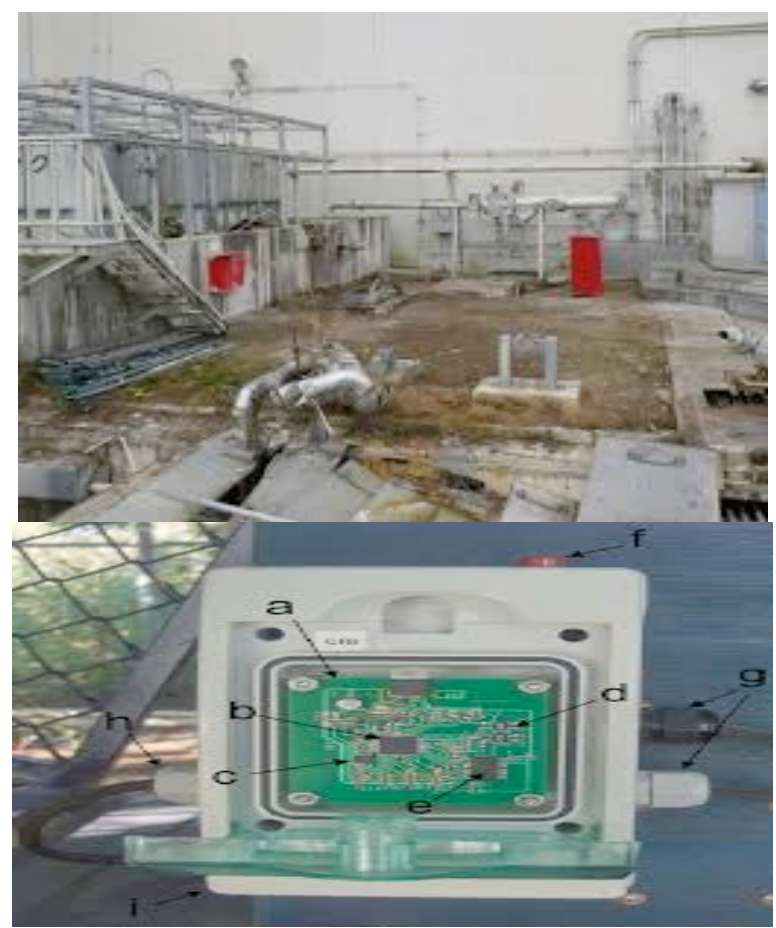

Figure 3. Wireless sensor applications in nuclear power plants 


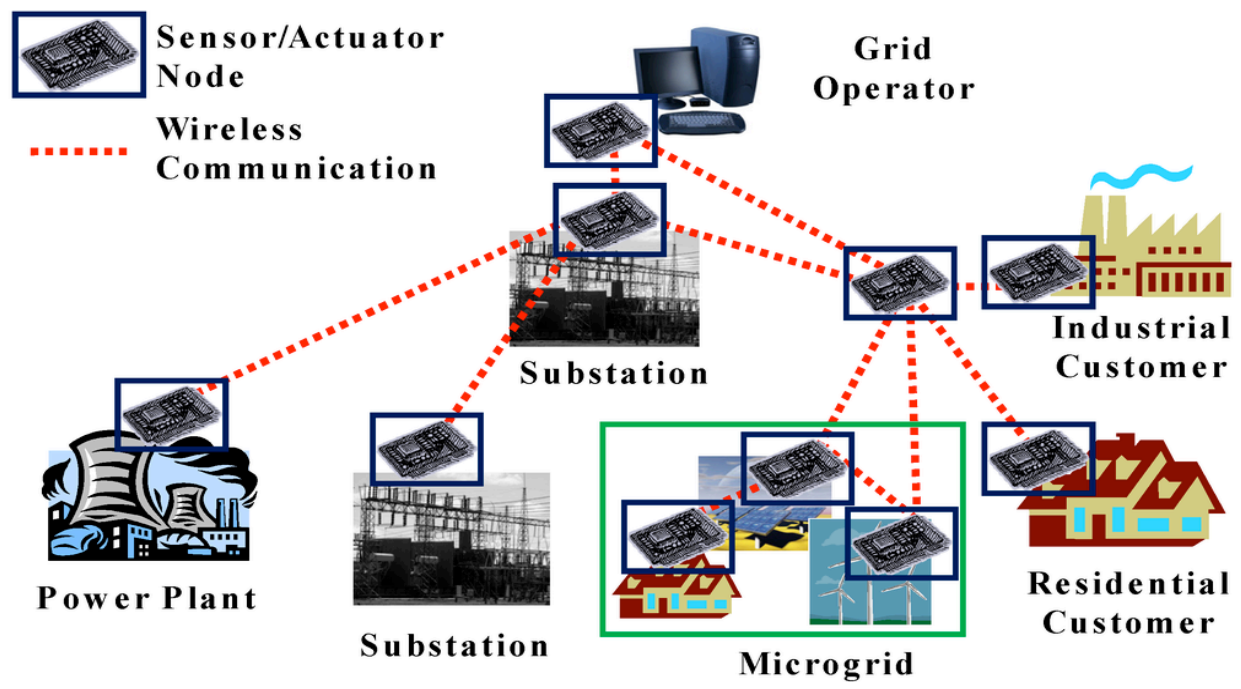

Figure 4. Nuclear power plant with wireless sensor operating mode

\section{Thermal Instrumentation Calibration Device Overview}

A thermal instrumentation calibration device is a special measuring instrument for verifying and calibrating various working thermocouple industrial thermal resistance, expansion thermometer temperature instrumentation, and various temperature sensors and transmitter [8]. The proposed device is compatible with the computer system, can automatically adjust the temperature measurement, access control, acquire and process data, and report output in accordance with the test procedures and certificates [9] (See figure 5).

\section{Hardware Design}

In the current study, the most common thermocouple calibration and thermal resistance test draws the basic structure of the figure 6 .

The thermal test device is used as follows.

The main function of this device is controlling the operation of the entire verification equipment and real-time communication with digital multi. An external controller, namely, RS232, is used to control the scanner [10]. In this case, the controller uses the S3C2410 industrial control panels and Windows CE 5.0 operating system, which is the control software for the specific test procedures. The scanner, stepping motor, and low potential scan switch constitute the main functions controlled by the microcontroller. These functions enable the internal DMM measurement channels in standard thermocouple/RTD and thermocouples plurality of subjects.

Between / heat resistance in order to switch, which is played thermocouple test procedures in the role of multipoint switch, operation of the.

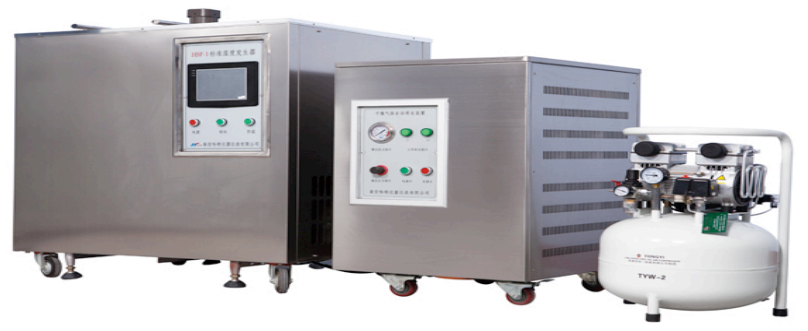

Figure 5. Thermal instrumentation calibration device

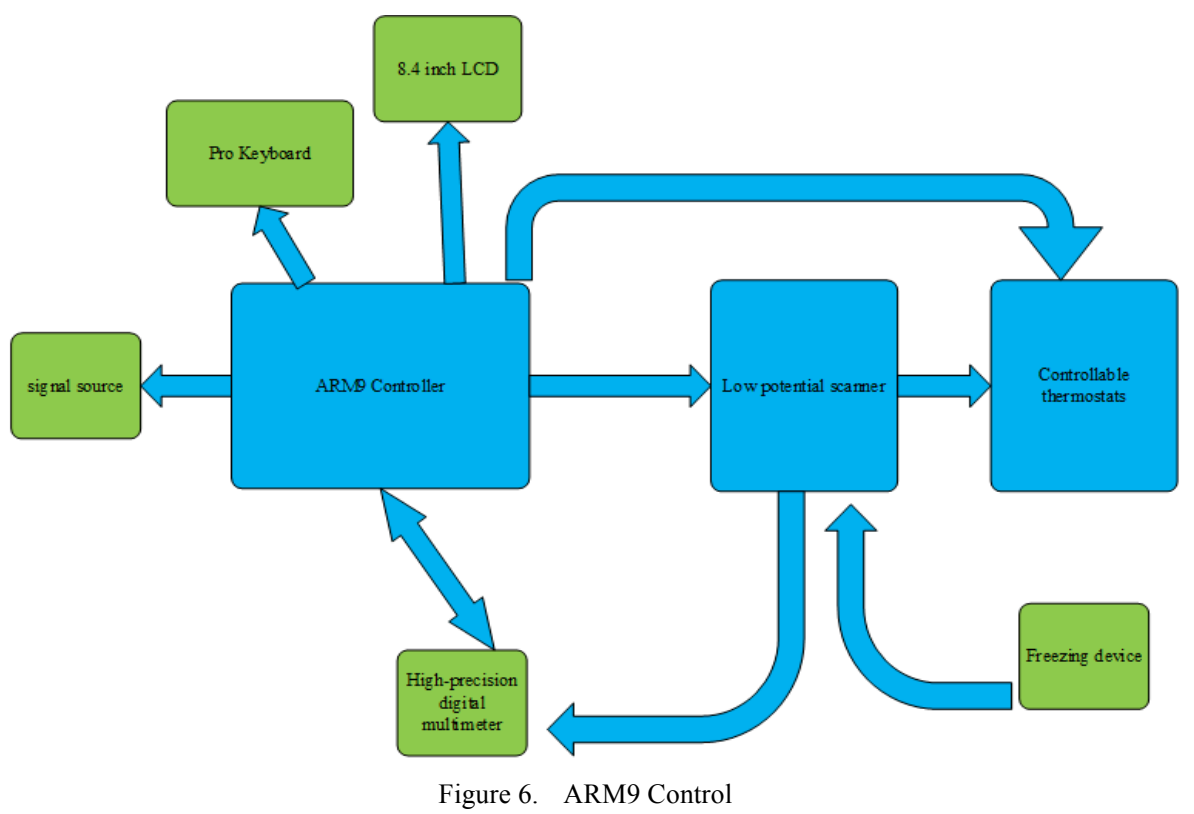




\section{E. Software Design}

The software design mainly includes three parts. First, the PLATFUN 2 Builder 5.0 is suitable for the production of industrial ARM9 board, WINDOWS CE 5.0 operating system image is used, and commissioning is conducted to achieve its embedding. Second, the use of EVC realizes transplantation on the PC software. This will not only avoid the computer system to bring all. The bad image can also make the design of the entire device considerably systematic and professional $[11,12]$. This design approach is the first case in the field of domestic metering. Finally, part of the source signal microcontroller program design and analyze the operating system $[13,14]$. The source of the problem solving nonlinear least squares method using MATBAL software programming and obtained correction equation. In addition, the design process will be used once the algorithm least squares details are provided. The nonlinear compensation formula is as follows:

$$
y(k)=h^{T}(K) \theta+e(k)
$$

where $y(K)$ and $h^{T}(K)$ are the observable data, the value of which can be obtained by conducting an experiment; $\mathrm{p}$ is the parameter to be estimated; and $e(k)$ is the error. Using the least squares method, the parameters to be estimated by the value of seconds should seek to make a difference to the square each time the observed and calculated values of the metric value is multiplied by its accuracy and minimum $[15,16]$. Therefore, we take the following criterion function:

$$
Z(\theta)=\sum_{k=1}^{n}[e(k)]^{2}=\sum_{k=1}^{n}\left[y(k)-h^{T}(K) \theta\right]^{2}=\left(Y_{L}-H_{L} \theta\right)^{T}\left(Y_{L}-H_{L} \theta\right)
$$

$$
\frac{\partial Z(\theta)}{\partial \theta}=\frac{\partial}{\partial \theta}\left(Y_{L}-H_{L} \theta\right)^{T}\left(Y_{L}-H_{L} \theta\right)=0
$$

We expand the formula and apply Equations (4) and (5):

$$
\begin{array}{r}
\frac{\partial}{\partial X}\left(a^{T} x\right)=a^{T} \\
\frac{\partial}{\partial X}\left(X^{T} A X\right)=2 X^{T} A
\end{array}
$$

where $\mathrm{A}$ is a symmetric matrix into a canonical equations as follows:

$$
\left(H_{L}^{T} H_{L}\right)^{-1} \theta=H_{L}^{T} Y_{L}
$$

when $\left(H_{L}^{T} H_{L}\right)$ is a regular matrix

$$
\theta=\left(H_{L}^{T} H_{L}\right)^{-1} H_{L}^{T} Y_{L}
$$

and

$$
\frac{\partial^{2} Z(\theta)}{\partial \theta^{2}}=2 H_{L}^{T} H_{L}>0 .
$$

When $\theta=\left(H_{L}^{T} H_{L}\right)^{-1} H_{L}^{T} Y_{L}, Z(\theta)$ is least and only.

\section{Result AnAlysis And Discussion}

From the requirements of the test procedures, debugging the source requires the use of numerous bits than its digital multi $[17,18]$. Using KEITHLEY $2000(61 / 2-$ resolution) digital form as a detection device during debugging, the median topic design source is determined to be five and a half. Given that the thermocouple is analog, the voltage signal simulation process is essentially based on thermal resistance $[19,20]$; thus, the analog voltage level of accuracy should be measured first. Several problems are encountered during the analog voltage output: the dynamic response of the output value is not fast enough, changes cannot be immediately followed, and considerable delay in the output signal. Thereafter, we determined that a few of the lower order filter source capacitors use ordinary chip capacitors. However, if the pressure is generally low, then it will replace all the polypropylene film capacitors after the problem is solved. On the left is the signal source and the right is the KEITHLEY2000 digital multi. At this time, the current source output is displayed at $1.00000 \mathrm{~V}$. The KEITHLEY2000 digital table shows that the actual output measured value is $1.00001 \mathrm{~V}$. Thus, the accuracy of the signal source is high. In addition, the debugging process temperature coefficient signal sources are tested; the test temperature coefficient is constant and humidity box cover. At $5{ }^{\circ} \mathrm{C}$. Range of $45^{\circ} \mathrm{C}$, the output voltage does not change more than five words, far exceeding the design requirements (in the range hood, the output voltage changes $5{ }^{\circ} \mathrm{C}-45^{\circ} \mathrm{C}$ not more than 20 words). Therefore, the design of the signal source is successful. At the end of the verification, the program will automatically check the results stored in the database table that is equally

TABLE I.

THE LIST OF EXAMINING

\begin{tabular}{|l|l|l|l|}
\hline Check number & \multicolumn{1}{|c|}{ Meter } & \multicolumn{1}{c|}{ Personnel } & \multicolumn{1}{c|}{ Result } \\
\hline JYJL000000 & YL0001 & Automatically check & Qualified \\
\hline JYJL000001 & YL0001 & Automatically check & Qualified \\
\hline JYJL000002 & YL0001 & Automatically check & Qualified \\
\hline JYJL000003 & YL0001 & Automatically check & Qualified \\
\hline
\end{tabular}

\section{CONCLUSION}

This study mainly discusses the wireless sensor applications in nuclear power plants and application of thermal instrumentation calibration device based on an embedded system. To solve the current problem of thermal instrumentation calibration device, a professional embedded computer system is used to replace the traditional version. Currently, the PC operating system from the desktop Windows CE operating system should be changed, and the PC software porting and optimization should be completed. By maximizing the highly specific and embedded computer technology, we can easily and safely control the operation of nuclear power plants. 


\section{REFERENCES}

[1] Wu, Hsi-Chin, et al., "Development and Characterization of a Bioinspired Bone Matrix with Aligned Nanocrystalline Hydroxyapatite on Collagen Nanofibers," Materials, 2016, vol. 9, no.3, pp. 198. http://dx.doi.org/10.3390/ma9030198

[2] Thompson, David S., et al., "Scoping review of complexity theory in health services research," BMC Health Services Research, 2016 , vol. 16, no.1, pp. 1. http://dx.doi.org/10.1186/s12913-016-1343-4

[3] Yadav, Ashish, et al., "Spectral plasmonic effect in the nanocavity of dye-doped nanosphere-based photonic crystals," Nanotechnology, 2016, vol. 27, no.16, pp. 165703. http://dx.doi.org/10.1088/0957-4484/27/16/165703

[4] Timofeev, Viktor N., "Control of convective flows in liquid metal in fluenced," Engineering \& Technologies, 2015, vol. 5, no.1, pp. 28-38.

[5] Chen, Xiaoshuang, et al., "A multivariate grey RBF hybrid model for residual useful life prediction of industrial equipment based on state data," International Journal of Wireless and Mobile Computing, 2016, vol. 10, no.1, pp. 90-96. http://dx.doi.org/10.1504/ IJWMC.2016.075230

[6] Kuo, P. H., "A glance at FD-MIMO technologies for LTE," IEEE Wireless Communications, 2016, vol. 23, no.1, pp. 2-5. http://dx.doi.org/10.1109/MWC.2016.7422396

[7] Li, Feng, Lei Ju, and Zhiping Jia., "Data aggregation framework for energy-efficient WirelessHART networks," Journal of Systems Architecture, 2016, vol. 63, pp. 70-79. http://dx.doi.org/10.1016/ j.sysarc.2016.01.009

[8] Lin, Feilong, et al., "A separate design principle for priority-aware packet collection in industrial cyber-physical systems," EURASIP Journal on Wireless Communications and Networking, 2016, vol. 1, pp. 1-14. http://dx.doi.org/10.1186/s13638-016-0572-x

[9] Shahzad, Aamir, et al., "A Secure, Intelligent, and Smart-Sensing Approach for Industrial System Automation and Transmission over Unsecured Wireless Networks," Sensors, 2016, vol. 16, no.3, pp. 322. http://dx.doi.org/10.3390/s16030322

[10] Ahuja, A. K., R. Chakka, and T. S. Pathak., "First Order Correlations in Simulated and Real Digital Traffic: Importance, Study \& Analysis," Indian Journal of Scientific \& Industrial Research, 2016, vol. 75, pp. 159-171.

[11] Ahrens A, Mcgrath C, Hagg U. "Subjective efficacy of oral appliance design features in the management of obstructive sleep apnea: A systematic review". American Journal of Orthodontics and Dentofacial Orthopedics, 2010, vol. 138, no.5, pp. 559-576. http://dx.doi.org/10.1016/j.ajodo.2010.01.030

[12] Liang, C. \& Razavi, B. "Systematic Transistor and Inductor Modeling for Millimeter-Wave Design". IEEE Journal of Solid- state Circuits, 2009, vol. 44, no. 2, pp.450-457. http://dx.doi.org/10.1109/JSSC.2008.2011031

[13] Koutroulis E, Kalaitzakis K, Voulgaris N C. "Development of a microcontroller-based, photovoltaic maximum power point tracking control system". Power Electronics, IEEE Transactions on, 2001, vol. 16, no. 1, pp. 46-54. http://dx.doi.org/10.1109/ 63.903988

[14] Gay D, Levis P, Von Behren R, et al. "The nesC language: A holistic approach to networked embedded systems" Acm Sigplan Notices. ACM, 2003, vol. 38, no. 5, pp.1-11. http://dx.doi.org/10.1145/780822.781133

[15] Nakajima N, Tamura T, Yamanishi Y. Network "Completion Using Dynamic Programming and Least-Squares Fitting”. The Scientific World Journal, 2012, pp. 957620-957620. http://dx.doi.org/10.1100/2012/957620

[16] Yingjie Z, Liling G. "Improved moving least squares algorithm for directed projecting onto point clouds". Measurement, 2011, vol. 44, no. 10, pp.2008-2019. http://dx.doi.org/10.1016/j.measure ment.2011.08.015

[17] Shabra A, Lee H. "Oversampled pipeline A/D converters with mismatch shaping”. IEEE Journal of Solid-state Circuits, 2002, vol. 37, no. 5, pp. 566-578. http://dx.doi.org/10.1109/4.997849

[18] Sasaki T, Yamamoto Y, Koashi M. "Practical quantum key distribution protocol without monitoring signal disturbance". Nature, 2014, pp. 509(7501).

[19] Efremov R, Volynsky P, Nolde D. "Monte Carlo simulations of voltage-driven translocation of a signal sequence". FEBS Letters, 2002, vol. 526, no. 1, pp.97-100. http://dx.doi.org/10.1016/S00145793(02)03145-9

[20] Zhou W, Khaliq A, Tang Y. "Simulation and design of piezoelectric microcantilever chemical sensors". Sensors and Actuators Aphysical, 2005, vol. 125, no. 1, pp.69-75. http://dx.doi.org/10. $\underline{1016 / j . s n a .2005 .07 .009}$

\section{AUTHORS}

Zhe YANG is with the Suzhou Nuclear Power Research Institute, Shenzhen, Guangdong, China (e-mail: ycengr@126.com).

Cheng YANG is with the Suzhou Nuclear Power Research Institute, Shenzhen, Guangdong, China (e-mail: chyeng-r@163.com).

Submitted, February, 17, 2016. Published as resubmitted by the authors on May, 16, 2016. 